\title{
An Open-Label, Multinational, Multicenter, Phase IIlb Study with Subcutaneous Administration of Trastuzumab in Patients with HER2-Positive Early Breast Cancer to Evaluate Patient Satisfaction
}

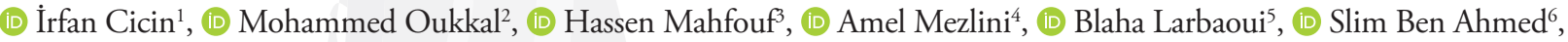

(1) Hassan Errihani ${ }^{7}$, (1) Khalid Alsaleh ${ }^{8}$, (1) Rhizlane Belbaraka ${ }^{9}$, (1) Perran Fulden Yumuk ${ }^{10}$, (1) Burce Goktas ${ }^{11}$,

(1) Mustafa Özgüroğlu ${ }^{12}$

${ }^{1}$ Department of Medical Oncology, Trakya University Faculty of Medicine, Edirne, Turkey

${ }^{2}$ Department of Medical Oncology, Beni Messous University Hospital, Beni Messous, Algeria

${ }^{3}$ Centre Hospitalo-Univerisitaire de Rouiba, Service D’oncologie Médicale, Rouiba, Algeria

${ }^{4}$ Service de Médecine Carcinologique, Institut Salah Azaïz, Tunis, Tunisia

${ }^{5}$ Service D’oncologie Médicale, EHS Oncologie Emir Abdelkader Oran, Oran, Algeria

${ }^{6}$ Deparment of Oncology, Farhat Hached Hospital, Sousse, Tunisia

${ }^{7}$ Department of Oncology, National Institute of Oncology, Mohammed V University, Rabat, Morocco

${ }^{8}$ College of Medicine, Department of Medicine, King Saud University, Riyadh, Saudi Arabia

${ }^{9}$ Cadi Ayyad University, Faculty of Medicine, Medical Oncology Department, Marrakech, Morocco

${ }^{10}$ Deparment of Medical Oncology, Marmara University School of Medicine, Marmara University Pendik Training and Research Hospital, İstanbul, Turkey

${ }^{11}$ Roche Müstahzarları Industry Inc., İstanbul, Turkey

${ }^{12}$ İstanbul University-Cerrahpasa Cerrahpasa Medical Faculty, İstanbul, Turkey

\begin{abstract}
Objective: This study was designed to investigate treatment satisfaction in patients and Health Care Professionals (HCP) and to evaluate the safety and tolerability of subcutaneous (SC) trastuzumab in patients with human epidermal growth factor receptor 2 (HER2)-positive early breast cancer (eBC).

Materials and Methods: Two-hundred and twenty-three patients with eBC were screened, of whom 173 patients met the eligibility criteria and received at least one dose of SC trastuzumab. The primary efficacy endpoint was to assess patient satisfaction via a questionnaire.

Results: The majority of patients ( $\mathrm{n}=166,97.6 \%)$ reported satisfaction with the SC route. Patients and HCPs stated that SC trastuzumab was easy to use (93.5\% and 62.5\%, respectively) compared to the intravenous (IV) route and all HCPs ( $\mathrm{n}=16)$ expressed satisfaction with the SC route. Progression, disease recurrence or death was reported in 24 patients (13.8\%) by two years of follow up. Four-year disease-free survival (DFS) and overall survival (OS) rates were $84.2 \%( \pm 3.1)$ and $90.5 \%( \pm 4.7)$, respectively. A total of 1299 adverse events (AEs) were recorded over 4-years follow-up, nearly $97 \%$ of which were judged non-serious. The most common AEs were arthralgia $(n=54,4.2 \%)$, flu-like symptoms $(n=41,3.2 \%)$ and nausea $(n=39,3.0 \%)$. Fifty-four cardiac events, including left ventricular dysfunction, left ventricular failure and cardiotoxicity, were reported. Ejection fraction (EF) decrease [median decrease 3.5\% $(0.12-19.0)]$ was reported in $5.4 \%$ of cases. SC trastuzumab treatment was interrupted due to decreased EF in two cases.
\end{abstract}

Conclusion: SC trastuzumab was widely acceptable to both patients and HCPs. The safety and tolerability of SC trastuzumab was consistent with the known safety profile of SC and IV administration.

Keywords: subcutaneous, trastuzumab, breast cancer, HER2, patient satisfaction

Cite this article as: Cicin I, Oukkal M, Mahfouf H, Mezlini A, Larbaoui B, Ben Ahmed S, Errihani H, Alsaleh K, Belbaraka R, Yumuk PF, Goktas B, Özgüroğlu M. An Open-Label, Multinational, Multicenter, Phase IIIb Study with Subcutaneous Administration of Trastuzumab in Patients with HER2Positive Early Breast Cancer to Evaluate Patient Satisfaction. Eur J Breast Health 2022; 18(1): 63-73

\section{Key Points}

- SC injection of trastuzumab was easy and satisfactory for both patients and healthcare professionals.

- A high percentage of patients receiving adjuvant therapy preferred subcutaneous trastuzumab over intravenous administration.

- The safety and tolerability of subcutaneous trastuzumab was consistent with the known safety profile for subcutaneous and intravenous administration 


\section{Introduction}

Breast cancer $(\mathrm{BC})$ is the most common cancer diagnosed among women and is the fifth leading cause of cancer deaths worldwide (1). Globally, there were 2.1 million new BC cases and 626,679 BC-related deaths reported in 2018, representing nearly $11.6 \%$ of all new cancer cases and $6.6 \%$ of cancer-related deaths.

Approximately $20 \%$ of BCs are characterized by amplification and/or overexpression of human epidermal growth factor receptor 2 (HER2, also called ErbB2), which is associated with poor prognosis and survival $(2,3)$. Trastuzumab, a humanized monoclonal antibody that targets and binds the HER2 protein, was approved by the US Food and Drug Administration (FDA) for therapeutic use in cases of metastatic BC in 1998 and HER2-positive eBC in $2006(4,5)$. Various randomized trials have shown that adjuvant trastuzumab for HER2-positive eBC improved disease-free survival (DFS) and overall survival (OS) $(6,7)$. Currently, adjuvant treatment with trastuzumab is a well-established treatment in HER2-positive eBC.

Trastuzumab was first licensed in clinical practice as an intravenous (IV) formulation, and in August 2013 subcutaneous (SC) trastuzumab (Herceptin ${ }^{\circledast}$ SC), which contains a fixed dose of $600 \mathrm{mg} / 5 \mathrm{~mL}$ and recombinant human hyaluronidase $\mathrm{PH} 20$, was authorized by the European Medicines Agency (EMA) (8), providing a shorter administration duration of 2 to 5 minutes, while the IV formulation is administered as a 90-minute infusion (9). Besides clinical benefits and a good tolerability profile, SC trastuzumab treatment may be associated with cardiac toxicities such as congestive heart failure (CHF), which may require close monitoring of left ventricular ejection fraction (LVEF) in all patients before and during treatment (10).

The Hannah study indicated that SC trastuzumab was non-inferior to IV formulation with a similar safety profile, and the PrefHer study showed that SC trastuzumab was the preferred treatment option among patients and health care professionals over IV administration (11, 12). Considering the two equally safe formulations of trastuzumab, patients' and health care professionals' (HCP) preference may be related to the improvement in quality of life, ease of administration and overall satisfaction with SC trastuzumab treatment.

This study was conducted as part of a global umbrella study "UmbHER1", which consists of a family of multiple studies with similar design, including MetaspHER (13), BELIS (14), Schearly (15) and SAPPHIRE (16), to assess the safety and tolerability of trastuzumab solution injected subcutaneously [vial or single-use injection device (SID)] in patients with HER2-positive BC. The preference of patients and HCPs was evaluated by implementing an inhouse developed questionnaire for testing patient- and HCP-reported outcomes in terms of overall satisfaction and treatment experience with SC trastuzumab at hospitals in patients with HER2-positive eBC conducted as a daughter study of the umbrella program.

\section{Materials and Methods}

\section{Study design and patient population}

The ML28851 study is a Phase IIIb, open-label, multinational, multicenter study to assess patient satisfaction with, HCP experience, and safety and tolerability of trastuzumab solution injected subcutaneously (SC; vial) in patients with HER2-positive eBC in a (neo)adjuvant
Eligible patients were women and men aged 18 years or older with HER2-positive (immunohistochemistry 3+ or positive by in situ hybridization), histologically confirmed, non-metastatic primary invasive breast carcinoma with no evidence of residual, locally recurrent, or metastatic disease after completion of surgery and chemotherapy (neoadjuvant or adjuvant), an Eastern Cooperative Oncology Group (ECOG) performance status of 0 or 1 , and a baseline LVEF of $55 \%$ or more before the first dose of trastuzumab. HER2-positivity was assessed by local laboratories using a validated assay. Radiotherapy and/or hormone therapy were allowed. Patients could have been either trastuzumab-naïve or already received IV trastuzumab following neoadjuvant or adjuvant chemotherapy. All patients provided written informed consent. Approval for the protocol was obtained from the appropriate ethic s committees and regulatory authorities. This study was approved by the Ethics Committee of Hacettepe University, with the registration date: 03.10.2013/decision no: 2013/12-06 (KA-120089); (ClinicalTrials. gov Identifier: NCT01964391).

\section{Procedures}

SC trastuzumab was administered via a hand-held syringe over a period of 5 minutes with a fixed dose of $600 \mathrm{mg} / 5 \mathrm{~mL}$ (including 10,000 units $\mathrm{rHuPH} 20$; irrespective of body weight) throughout the study every 3 weeks ( $\mathrm{q} 3 \mathrm{w}$ ) for up to 18 cycles. Dose reductions were not permitted. For non-naïve patients, the duration of SC trastuzumab treatment was expected to be shorter.

The primary objective of the study was the assessment of patient satisfaction by an internally developed Patient Satisfaction Questionnaire (PSQ) that consisted of 20 questions. The secondary objectives were the assessment of the safety and tolerability of SC trastuzumab treatment along with the overall satisfaction of HCPs via an HCP Experience Questionnaire comprising 14 questions. Both questionnaires were completed at the end of the treatment period, and the questionnaires themselves have been added to the manuscript as supplementary material. For the assessment of survival, a follow-up period of up to four years was defined for the evaluation of overall survival (OS) and disease-free survival (DFS).

\section{Assessments}

All enrolled patients who received at least one dose of the study medication ( $S C$ trastuzumab) were included in the safety population, and adverse events (AEs) and serious adverse events (SAEs) were monitored and documented at each tri-weekly treatment visit and during the safety follow-up visits. AEs and SAEs were graded according to the National Cancer Institute Common Terminology Criteria for Adverse Events (NCI-CTCAE) 4.0; congestive heart failure (CHF) was classified using the New York Heart Association (NHYA) functional classification.

Cardiac function was monitored by measuring left ventricular ejection fraction (LVEF) using echocardiography or a multigated acquisition (MUGA) scan and electrocardiogram (ECG), and assessment of cardiac signs and symptoms. Cardiac safety assessments were performed at screening visits, approximately every three months during SC trastuzumab treatment, during the safety follow-up visits, and then at 6,12 and 24 months after treatment cessation.

\section{Statistical Analysis}

The sample size calculation was based on the patient satisfaction rate defined for subcutaneous trastuzumab (75\%), and to achieve adequate 
power, a minimum 162 patients should be enrolled. Descriptive statistical methods were used to analyze and report the results of this single-arm study. Patient satisfaction was evaluated in the intentionto-treat (ITT) population who completed the PSQ $(\mathrm{n}=171)$. HCP treatment satisfaction was evaluated among responding investigators $(\mathrm{n}=16 / 30)$. Safety analyses were conducted in patients who received at least one dose of SC trastuzumab $(n=173)$, and AEs were graded per standard criteria. The Kaplan-Meier method was used to estimate the median DFS and OS. Hazard ratios (HRs) from timewise comparisons of results were estimated by Cox proportional hazards regression. Statistical analyses were performed with the Stata software (version 10.0).

\section{Results}

\section{Study Population and Demographics}

Between February 21, 2014, and November 12, 2018 (data cut-off date), a total of 174 patients (ITT population) were randomly assigned by 30 HCPs at 25 centers in Turkey, Algeria, Saudi Arabia, Morocco, and Tunisia. One patient was excluded due to violation of eligibility criteria after randomization. One hundred and seventy-three patients received at least one valid dose of SC trastuzumab and comprised the safety population. At the time of data cut-off, 148 patients $(85.5 \%)$ had completed treatment as per protocol and were alive (Figure 1).

The median (range) age of patients was 49.5 (28-86) years. Left-breast cancer was more frequent $(52.9 \%)$, and $13.2 \%$ had previously received radiotherapy before the initiation of trastuzumab treatment. The mean $( \pm$ SD) primary tumor size was $35.3( \pm 29.1) \mathrm{mm}$ (range: $2.1-250$
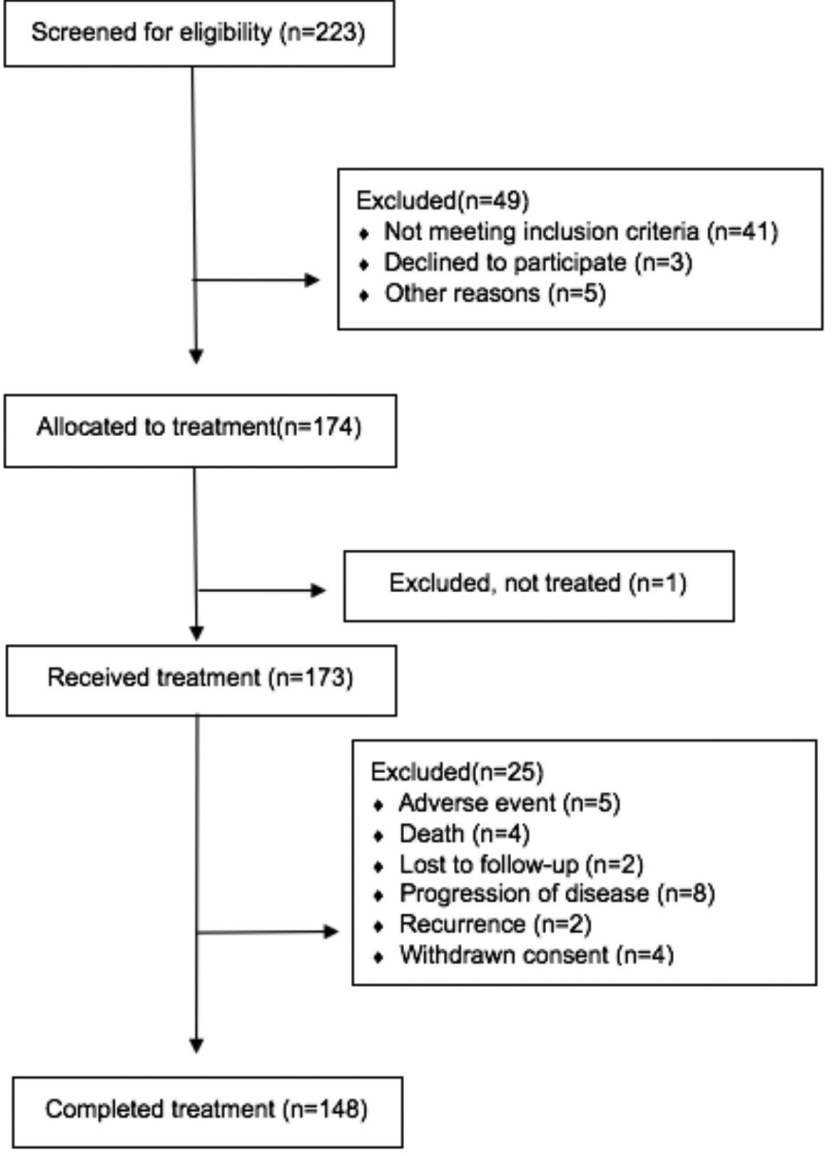

Figure 1. Trial profile $\mathrm{mm})$ in terms of maximum diameter, and $162(93.1 \%)$ patients were diagnosed with ductal carcinoma. Baseline demographics and tumor characteristics are shown in Table 1.

Table 1. Baseline demographics and tumor characteristics

\section{Variable}

$(n=174)$

\section{Age (years)}

Mean (SD)

Median (min-max)

$49.5(28-86)$

Sex, $n$ (\%)

Female

173 (99.4)

Male

$1(0.6)$

Race, $\mathbf{n}$ (\%)

Caucasian

$172(98.9)$

Black

Time since initial diagnosis (months)

Mean (SD)

$7.1(3.9)$

Median (min-max)

$6.9(0.3-20.8)$

Location of primary tumor, $\mathbf{n}$ (\%)

Left

Right

79 (45.4)

Bilateral

Distribution of primary tumor, $\mathbf{n}(\%)$

Unifocal

$133(76.4)$

Multifocal

Multicentric

6 (3.4)

Tumor size

Primary tumor size (largest diameter, $\mathrm{mm}$ )

Mean (SD)

Multiple foci (largest diameter, $\mathrm{mm}$ )

Mean (SD)

Breast Cancer Subtype, n (\%)

Ductal

$162(93.1)$

Lobular

Other

Missing

$2(1.1)$

Histological grade, n (\%)

Moderately differentiated

Poorly differentiated

Unknown

75 (43.1)

Well differentiated

24 (13.8)

Nuclear grade, n (\%)

Grade 1

Grade 2

Grade 3

$63(36.2)$

Unknown

$32(18.4)$ 
Table 1. Continued

\begin{tabular}{|c|c|}
\hline Variable & $(n=174)$ \\
\hline \multicolumn{2}{|c|}{ HER2 status IHC, n (\%) } \\
\hline $2+$ & $11(6.3)$ \\
\hline $3+$ & $160(92.0)$ \\
\hline Missing & $3(1.7)$ \\
\hline \multicolumn{2}{|c|}{ HER2 status FISH, n (\%) } \\
\hline Positive & $91(98.9)$ \\
\hline Negative & $1(1.1)$ \\
\hline \multicolumn{2}{|c|}{ ECOG performance status, n (\%) } \\
\hline 0 & $148(85.1)$ \\
\hline 1 & $26(14.9)$ \\
\hline \multicolumn{2}{|c|}{ Type of prior anti-cancer therapy, n (\%) } \\
\hline Chemotherapy & $122(70.1)$ \\
\hline Hormonal therapy & $40(23.0)$ \\
\hline Radiotherapy & $46(26.4)$ \\
\hline Unknown & $5(2.9)$ \\
\hline \multicolumn{2}{|c|}{ Current treatment status, n (\%) } \\
\hline eBC adjuvant & 139 (79.9) \\
\hline eBC neo-adjuvant & $35(20.1)$ \\
\hline \multicolumn{2}{|c|}{$\begin{array}{l}\text { SD: Standard deviation, min: Minimum, max: Maximum, } \\
\text { HER2: Human epidermal growth factor receptor 2; IHC: } \\
\text { Immunohistochemistry, FISH: Fluorescence in situ hybridization, } \\
\text { ECOG: Eastern Cooperative Oncology Group, eBC: Early breast } \\
\text { cancer, n: Number }\end{array}$} \\
\hline
\end{tabular}

\section{Patient Preference}

A total of 170 patients completed the PSQ, and 97.6\% ( $\mathrm{n}=166$ ) reported satisfaction with administration via the SC route; only $2.4 \%$ reported dissatisfaction. The experience with SC trastuzumab was reported as "acceptable" by $95.6 \%$ of patients and "fairly unpleasant" by $4.1 \%$ of patients. The majority of patients $(93.5 \%)$ revealed that the medical or nursing staff administering SC trastuzumab did not experience any difficulty during the infusion.

Patient satisfaction results showed that SC trastuzumab administered via handheld syringe caused no bruising, irritation, or infection around the injection site $(67.6 \%, 75.9 \%$ and $91.8 \%$ of patients, respectively). Administration time was reported as less than 5 minutes by $76.5 \%$ of patients and 6-10 minutes by $22.9 \%$ of patients. Subcutaneous administration was described as painless by $66.5 \%$ of patients, painful by $30.6 \%$, and very painful by $5.6 \%$ of patients. Three-quarters of patients $(75.9 \%)$ did not experience any adverse reactions such as bruising or irritation during or after SC administration, and $75.6 \%$ of patients did not feel anxious during SC treatment. The questionnaire also tried to define the burden of attending a health care center for injections, with items on ease of travelling, time spent and costs. The results showed that attending the clinics required some effort on the part of at least $25 \%$ of patients, with a minimum public transportation time of over one hour $(100 \%)$, and all patients had to be escorted by another person $(58.5 \%)$. The questionnaire and outcomes of patient responses are presented in Appendix 1 - Patient Satisfaction

\section{HCP Preference}

Approximately half of HCPs completed the treatment satisfaction questionnaire about their preferences $(n=16,62.5 \%$ were principal investigators). All respondents were satisfied with SC trastuzumab. Responses demonstrated that $81.3 \%$ of HCPs would strongly recommend SC trastuzumab for their patients and $62.5 \%$ of HCPs reported SC administration was very easy, with the remainder reporting this administration route to be fairly easy.

The majority of HCPs found no bruising or infection caused by SC infusion around the injection site $(81.3 \%$ and $93.8 \%$, respectively) whereas $56.3 \%$ reported a few occurrences of irritation. The time spent on preparation and administration was reported as less than 5 minutes by $43.8 \%$ and $75 \%$ of HCPs, respectively, and patient chair time per cycle was found to be between 3 and 4 hours. The questionnaire and outcomes of HCPs are given in Appendix 2 - Healthcare Professional Experience Questionnaire.

\section{Efficacy}

Survival analyses were performed for DFS and OS in 173 patients. DFS events - progression, recurrence of disease or death - were observed in 24 patients. Nine patients $(5.2 \%)$ died during a median follow-up time of $36.6( \pm 10.3)$ months [ $95 \%$ confidence interval $(\mathrm{CI})$ : 35.1-38.1]. One-hundred and forty-one patients were assessed for the survival analysis, while 23 patients were not subject to follow-up after withdrawing consent.

The median OS time was 54.1 months (95\% CI: 52.5-55.6). The 1- and 4-year OS rates were $99.4 \%( \pm 0.6)$ and $90.5 \%( \pm 4.7)$, respectively. The median DFS was 50.9 months (95\% CI: 48.8-53.0). The 1- and 4-year DFS rates were $97.1 \%( \pm 1.3)$ and $84.2 \%( \pm 3.1)$, respectively (Figure 2$)$.

\section{Safety and Tolerability}

During the 4-year follow-up, 1,299 AEs were documented in 160 patients. There were 261 AEs defined as related to SC trastuzumab in 104 patients. There were 43 SAEs reported in 27 patients. Seven SAEs were evaluated as related to trastuzumab (Table 2). Ejection fraction decrease $(5.4 \%)$, injection site erythema $(5.0 \%)$, pain $(5.0 \%)$ and rash $(5.0 \%)$ were the most common treatment-related AEs.

Five patients experienced AEs leading to permanent discontinuation of the study treatment. Two patients experienced grade 2 and grade 3 LVEF declines, and one patient experienced a SAE of grade 3 cardiotoxicity that led to discontinuation of the study treatment, whereas the remainder had grade 2 peripheral motor neuropathy and erythema. All events remained unresolved.

Twelve patients experienced 17 AEs that required a dose modification/ temporary interruption of the study treatment, mostly due to ejection fraction decrease.

\section{Cardiac Adverse Events}

Forty-two patients $(24.2 \%)$ experienced 76 cardiac-associated AEs of any grade, possibly related to treatment in 40 cases. Nineteen patients developed $21(27.6 \%)$ events of trastuzumab-induced symptomatic left ventricular systolic dysfunction (LVSD), of which 15 events were recorded as AEs and six events as SAEs. Fifty-five events of asymptomatic LVSD were recorded in 32 patients, all of which were reported as AEs (Table 3).

Twenty-eight adverse events ( 24 considered as treatment-related) were recorded as decreased LVEF (including ejection fraction decrease, left 
a) $\mathrm{OS}$

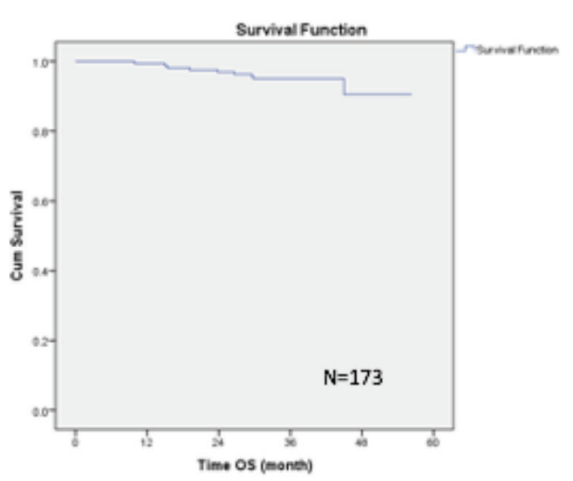

\begin{tabular}{|c|c|c|c|}
\hline Total (n) & Number of & \multicolumn{2}{|c|}{ Censored } \\
& events & $\mathrm{N}$ & $\%$ \\
\hline 173 & 9 & 164 & 94.8 \\
\hline
\end{tabular}

b) DFS

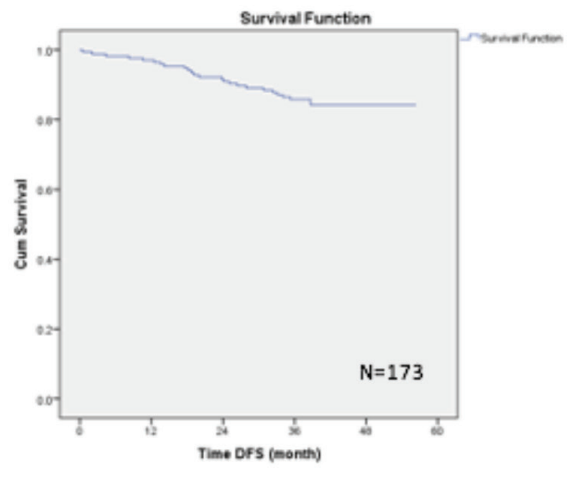

\begin{tabular}{|c|c|c|c|}
\hline Total (n) & Number of & \multicolumn{2}{|c|}{ Censored } \\
& events & $\mathrm{N}$ & $\%$ \\
\hline 173 & 24 & 149 & 86.1 \\
\hline
\end{tabular}

Kaplan-Meier - Cumulative Proportion Surviving at Cut-off

\begin{tabular}{|c|c|c|}
\hline & Proportion & Std. Enror \\
\hline 1. year & $99.4 \%$ & $0.6 \%$ \\
\hline 2. year & $98.7 \%$ & $0.9 \%$ \\
\hline 3. year & $95.0 \%$ & $1.7 \%$ \\
\hline 4. year & $90.5 \%$ & $4.7 \%$ \\
\hline
\end{tabular}

Figure 2. Overall survival (OS) and disease-free survival (DFS) curves

\begin{tabular}{|c|c|c|}
\hline & Proportion & Std. Error \\
\hline 1. year & $99.4 \%$ & $0.6 \%$ \\
\hline 2. year & $98.7 \%$ & $0.9 \%$ \\
\hline 3. year & $95.0 \%$ & $1.7 \%$ \\
\hline 4. year & $90.5 \%$ & $4.7 \%$ \\
\hline
\end{tabular}

Table 2. Adverse events

\section{Adverse event category}

Incidence $\mathbf{n}(\%)$

Any $A E, n(\%)$

$1299(100)$

Non-serious $A E$, n (\%)

1256 (96.7)

AEs related to study treatment

$254(19.6)$

AEs leading to permanent discontinuation of study treatment

$4(0.3)$

AEs leading to dose modification/interruption

12 (0.9)

AEs of suspected cardiac origin

SAE, n (\%)

$43(3.3)$

SAEs related to study treatment

$7(0.5)$

Fatal SAEs

$3(0.2)$

Fatal SAEs related to study treatment

SAEs leading to permanent discontinuation of study treatment

SAEs leading to dose modification/ interruption

AE: Adverse event, SAE: Serious adverse event, n: Number Significant values are shown in bold.

ventricular dysfunction and failure, systolic and diastolic dysfunction, cardiotoxicity) in 23 patients (Table 4). The decline in median LVEF was found to be $3.5 \%(0.12-19.0)$. No fatal cardiac adverse events were reported.

\section{Discussion}

The ML28851 study is a prospective study to assess the impact of SC trastuzumab on the treatment experience and satisfaction of patients and HCPs, as well as the safety and tolerability of trastuzumab SC administered at healthcare centers. The study followed patients for a period of four years to define survival (OS and DFS) rates. Patients and HCPs voluntarily completed questionnaires developed by the investigators, and the majority of patients rated SC administration of trastuzumab as acceptable and satisfactory. Although only half of the HCPs completed the treatment satisfaction questionnaire, their responses indicated a strong rate of recommendation for SC trastuzumab for their patients.

Only a limited number of studies showing the preference of both patients and HCPs relating to long-term treatments, such as treatment of early breast cancer, have been published. The PrefHer study was the first clinical trial to assess patient preference for subcutaneous or intravenous administration of trastuzumab, as well as the satisfaction of healthcare professionals for the adjuvant treatment of HER2-positive early breast cancer (12). The MetaspHer study was conducted in a setting of metastatic disease and was designed to evaluate the preference of patients and HCPs between SC and IV administrations (13).

Our study results were consistent with those of these previous studies, wherein a high percentage of patients preferred SC trastuzumab over IV administration $(12,13)$. Despite the compatible results from these studies, the patient population enrolled in each study was different; the ML28851 study included patients with eBC in adjuvant and neoadjuvant settings. However, it should be noted that in the PrefHer study, SC trastuzumab was administered with a handheld syringe or SC injection device, whereas in our study the administration was performed with a syringe alone.

An important aspect of the study was the exploration of patient burden through an internally developed questionnaire for patients. The use of a SC injection of trastuzumab may provide the option of patient home care/treatment, as there is increasing demand among the patients for treatment in more comfortable treatment settings, or even at home. In a recent prospective study (BELIS) conducted in Belgium and Israel, the safety and patient experience of SC trastuzumab was 
evaluated. The study results showed that the safety profile was similar to in-patient treatment, and patients almost always preferred home administration (14).

In a study conducted in Germany, 70\%-90\% of patients preferred SC administration of trastuzumab and stated that the main reason for SC preference was time saved during administration (17). Based on the results of our study, the administration time of SC trastuzumab was reported to be less than 5 minutes by $76.5 \%$ of patients and 6 to 10 minutes by $22.9 \%$ of patients. Patients receiving IV trastuzumab

Table 3. Cardiac adverse events

\section{Adverse Event Category Cardiac AEs}

Any cardiac $A E, n$ (\%)

76 (100)

Non-serious cardiac AE, n (\%)

70 (92.1)

Symptomatic LVSD

15 (19.7)

Serious Cardiac AE, n (\%)

6 (7.9)

Symptomatic LVSD

$6(7.9)$

AEs associated with decreased LVEF

Any cardiac $A E, n$ (\%)

28 (100)

Non-serious cardiac $A E, n$ (\%)

25 (89.3)

AEs related to study treatment

$21(75.0)$

AEs leading to permanent discontinuation of study treatment

$2(7.1)$

AEs leading to dose modification/ interruption

Serious Cardiac AE, n (\%)

Serious AEs related to study treatment

SAEs leading to permanent discontinuation of study treatment

SAEs leading to dose modification/ interruption

AE: Adverse event; SAE: Serious adverse event; LVSD: Left ventricular systolic dysfunction; LVEF: Left ventricular ejection fraction Significant values are shown in bold.

Table 4. Adverse Events Associated with Decreased LVEF
Adverse Event Category AEs associated with decreased LVEF

Investigations

$16(57.1)$

Ejection fraction decreased

$16(57.1)$

Cardiac disorders

$12(42.9)$

Left ventricular dysfunction

8 (28.6)

Left ventricular failure

$1(3.6)$

Cardiotoxicity

1 (3.6)

Systolic dysfunction

$1(3.6)$

Diastolic dysfunction
AE: Adverse event; LVEF: Left ventricular ejection fraction, n: Number Significant values are shown in bold. spent more time in the oncology unit than those receiving SC administration. A UK time and motion study showed that the time dedicated to preparation and administration of SC trastuzumab by any HCP was three times shorter than in the case of IV trastuzumab on average (30.0 vs. 94.5 minutes) (18). The time spent by HCPs on preparation and administration in our study was less than 5 minutes for $\mathrm{SC}$ infusion in $75 \%$ of all cases, thus presenting a unique advantage in terms of ease of use.

Another reason for the preference for SC trastuzumab administration was reduced pain and discomfort around the injection site, consistent with previous clinical trials $(12,19)$. However, in several clinical studies investigating SC trastuzumab, the most common AE related to SC administration was injection-site reactions $(20,21)$.

Previous studies showed that trastuzumab-induced cardiotoxicity is independent of dose and notably reversible following treatment discontinuation (21-23). Therefore, AEs of suspected cardiac origin were strictly monitored during the treatment period, and the cardiac safety profile of SC trastuzumab was observed in this current study. The number of patients that experienced cardiac-associated AEs of any grade was similar to those observed in the Zambetti et al's (15) Schearly study but higher than in the HannaH and the PrefHer studies $(13,18)$. However, only one severe left ventricular failure was reported during this study. LVEF decrease $<55 \%$ was observed in $1.22 \%$ of the treatment population, and this decrease resolved completely during subsequent observation.

This study revealed that SC use of trastuzumab in eBC patients is a satisfactory treatment option, and indicated very high preference rates in patients and HCPs. These findings were also consistent with a recent prospective study showing that $\mathrm{SC}$ trastuzumab can be safely administered at home by an HCP, and patients clearly indicated such setting to be comfortable and overall beneficial.

Moreover, with a similar safety profile to that of the IV form, and with comparable OS and DFS rates to previous studies for patients with HER2-positive eBC $(11,24)$ our study confirmed the efficacy of the SC administration route. The data obtained from this study demonstrate that SC trastuzumab is a valid and preferred option for improving patients' and HCPs' satisfaction in cases of HER2-positive eBC.

The limitation of our study was the use of two separate, non-validated, internally developed questionnaires. However, the aim and outcomes of these questionnaires showed that non-validated questionnaires may serve as a reliable source of information if they contain calculable, objective information such as time, conditions or outcome information.

\section{Conclusion}

These results have shown that both patients and HCPs favored SC trastuzumab for the treatment of HER2-positive eBC. The safety and tolerability of SC trastuzumab is consistent with the known safety profile of SC and IV administration.

Acknowledgements: The authors thank İrem Ünsal of Monitor CRO for medical writing, editing, and reviewing services for this manuscript.

Ethics Committee Approval: This study was approved by the Ethics Committee of Hacettepe University, with the registration date: $03.10 .2013 /$ decision no: 2013/12-06 (KA-120089); (ClinicalTrials.gov Identifier: NCT01964391). 
Informed Consent: All patients provided written informed consent.

Peer-review: Externally peer-reviewed.

\section{Authorship Contributions}

Conflict of Interest: No conflict of interest declared by the authors.

Financial Disclosure: The authors declare that this study received no financial disclosure.

\section{References}

1. WHO Global Cancer Observatory. Breast cancer fact sheet Globocan 2018. Available at: https://gco.iarc.fr/today/data/factsheets/cancers/20Breast-fact-sheet.pdf [Crossref]

2. Mazzotta M, Krasniqi E, Barchiesi G, Pizzuti L, Tomao F, Barba M, et al. Long-term safety and real-world effectiveness of trastuzumab in breast cancer. J Clin Med 2019; 8: 254. (PMID: 30781624) [Crossref]

3. Wilson FR, Coombes ME, Wylie Q, Yurchenko M, Brezden-Masley C, Hutton B, et al. Herceptin (trastuzumab) in HER2-positive early breast cancer: protocol for a systematic review and cumulative network metaanalysis. Syst Rev 2017; 6: 196. (PMID: 29017563) [Crossref]

4. Hartkopf AD, Brendel MH, Wallwiener M, Taran FA, Brucker S, Grischke EM. Trastuzumab administration in patients with metastatic breast cancer - experience of a large university breast center. Geburtshilfe Frauenheilkd 2014; 74: 563-568. (PMID: 24976638) [Crossref]

5. Furrer D, Paquet C, Jacob S, Diorio C (2018). The human epidermal growth factor receptor 2 (HER2) as a prognostic and predictive biomarker: molecular insights into HER2 activation and diagnostic implications. 2018 Nov 5. doi: 10.5772/intechopen.78271. [Crossref]

6. Cameron D, Piccart-Gebhart MJ, Gelber RD, Procter M, Goldhirsch A, de Azambuja E, et al. 11 years' follow-up of trastuzumab after adjuvant chemotherapy in HER2-positive early breast cancer: final analysis of the HERceptin Adjuvant (HERA) trial. Lancet 2017; 389: 1195-1205. (PMID: 28215665) [Crossref]

7. Romond EH, Perez EA, Bryant J, Suman VJ, Geyer CE Jr, Davidson NE, et al. Trastuzumab plus adjuvant chemotherapy for operable HER2positive breast cancer. N Engl J Med 2005; 353: 1673-1684. (PMID: 16236738) [Crossref]

8. EMA Herceptin INN-trastuzumab summary of product characteristics 2014. Available at: https://www.ema.europa.eu/en/documents/productinformation/herceptin-epar-product-information_en.pdf [Crossref]

9. Dent S, Ammendolea C, Christofides A, Edwards S, Incekol D, Pourmirza B, et al. A multidisciplinary perspective on the subcutaneous administration of trastuzumab in HER2-positive breast cancer. Curr Oncol 2019; 26: e70-e80. (PMID: 30853812) [Crossref]

10. Joensuu H. Escalating and de-escalating treatment in HER2-positive early breast cancer. Cancer Treat Rev 2017; 52: 1-11. (PMID: 27866067) [Crossref]

11. Jackisch C, Stroyakovskiy D, Pivot X, Ahn JS, Melichar B, Chen SC, et al. Subcutaneous vs intravenous trastuzumab for patients with ERBB2-positive early breast cancer: final analysis of the HannaH Phase 3 randomized clinical trial. JAMA Oncol 2019; 5: e190339. (PMID: 30998824) [Crossref]

12. Pivot X, Gligorov J, Müller V, Curigliano G, Knoop A, Verma S, et al. Patients' preferences for subcutaneous trastuzumab versus conventional intravenous infusion for the adjuvant treatment of HER2-positive early breast cancer: final analysis of 488 patients in the international, randomized, two-cohort PrefHer study. Ann Oncol 2014; 25: 19791987. (PMID: 25070545) [Crossref]
13. Pivot X, Spano JP, Espie M, Cottu P, Jouannaud C, Pottier V, et al. Patients' preference of trastuzumab administration (subcutaneous versus intravenous) in HER2-positive metastatic breast cancer: Results of the randomized MetaspHer study. Eur J Cancer 2017; 82: 230-236. (PMID: 28648618) [Crossref]

14. Denys H, Martinez-Mena CL, Martens MT, D'Hondt RG, Graas ML, Evron E, et al. Safety and tolerability of subcutaneous trastuzumab at home administration, results of the phase IIIb open-label BELIS study in HER2-positive early breast cancer. Breast Cancer Res Treat 2020; 181: 97-105. (PMID: 32240454) [Crossref]

15. Zambetti M, Montemurro F, Morandi P, Zamagni C, Brandes AA, Bisagni G, et al. Safety profile of subcutaneous trastuzumab for the treatment of patients with HER2-positive early or locally advanced breast cancer: primary analysis of the SCHEARLY study. Eur J Cancer 2018; 105: 6170. (PMID: 30396014) [Crossref]

16. Woodward N, De Boer RH, Redfern A, White M, Young J, Truman M, Beith J. Results from the First Multicenter, Open-label, Phase IIIb Study Investigating the combination of pertuzumab with subcutaneous trastuzumab and a taxane in patients with HER2-positive metastatic breast cancer (SAPPHIRE). Clin Breast Cancer 2019; 19: 216-224. (PMID: 30922805) [Crossref]

17. Jackisch C, Müller V, Dall P, Neumeister R, Park-Simon TW, RufDördelmann A, et al. Subcutaneous trastuzumab for HER2-positive breast cancer - evidence and practical experience in 7 German centers. Geburtshilfe Frauenheilkd 2015; 75: 566-573. (PMID: 26166837) [Crossref]

18. Burcombe R, Chan S, Simcock R, Samanta K, Percival F, Barrett-Lee P. Subcutaneous trastuzumab (Herceptin ${ }^{\circ}$ ): a UK time and motion study in comparison with intravenous formulation for the treatment of patients with HER2-positive early breast cancer. Adv Breast Cancer Res 2013; 2: 133-140. [Crossref]

19. Gligorov J, Ataseven B, Verrill M, De Laurentiis M, Jung KH, Azim HA, et al. Safety and tolerability of subcutaneous trastuzumab for the adjuvant treatment of human epidermal growth factor receptor 2-positive early breast cancer: SafeHer phase III study's primary analysis of 2573 patients. Eur J Cancer 2017; 82: 237-346. (PMID: 28625777) [Crossref]

20. Ismael G, Hegg R, Muehlbauer S, Heinzmann D, Lum B, Kim SB, et al. Subcutaneous versus intravenous administration of (neo)adjuvant trastuzumab in patients with HER2-positive, clinical stage I-III breast cancer (HannaH study): a phase 3, open-label, multicentre, randomised trial. Lancet Oncol 2012; 13: 869-878. (PMID: 22884505) [Crossref]

21. Lazaro Cebas A, Cortijo Cascajares S, Pablos Bravo S, Del Puy Goyache Gońi M, Gonzalez Monterrubio G, Perez Cardenas MD, et al. Subcutaneous versus intravenous administration of trastuzumab: preference of HER2 + breast cancer patients and financial impact of its use. J BUON 2017; 22: 334-339. (PMID: 28534353) [Crossref]

22. Seidman A, Hudis C, Pierri MK, Shak S, Paton V, Ashby M, et al. Cardiac dysfunction in the trastuzumab clinical trials experience. J Clin Oncol 2002; 20: 1215-1221. (PMID: 11870163) [Crossref]

23. Swain SM, Ewer MS, Cortés J, Amadori D, Miles D, Knott A, et al. Cardiac tolerability of pertuzumab plus trastuzumab plus docetaxel in patients with HER2-positive metastatic breast cancer in CLEOPATRA: a randomized, double-blind, placebo-controlled phase III study. Oncologist 2013; 18: 257-264. (PMID: 23475636) [Crossref]

24. Earl HM, Hiller L, Vallier AL, Loi S, McAdam K, Hughes-Davies L, et al. 6 versus 12 months of adjuvant trastuzumab for HER2-positive early breast cancer (PERSEPHONE): 4-year disease-free survival results of a randomised phase 3 non-inferiority trial. Lancet 2019; 393: 2599-2612. (PMID: 31178152) [Crossref] 
Eur J Breast Health 2022; 18(1): 63-73

Appendix 1. Patient Satisfaction Questionnaire

\section{PATIENT SATISFACTION QUESTIONNAIRE}

Item Category

Frequency n (\%)

1. Where did you have your Herceptin study treatment?

Hospital chemotherapy department

$169(99.4)$

Other chemotherapy department

$1(0.6)$

Total

170

2. Was this the same place as for your chemotherapy treatment?

Yes

$157(92.4)$

No

$13(7.6)$

Total

170

3. How long did it take you to travel there?

$<1$ hour

$60(35.3 \%)$

$>2$ hours

47 (27.6\%)

1-2 hours

$63(37.1 \%)$

Total

170

4. How easy was it for you to travel there?

$\begin{array}{lr}\text { Fairly } & 83(48.8 \%) \\ \text { Not at all } & 46(27.1 \%) \\ \text { Very } & 41(24.1 \%)\end{array}$

Very

170

Total

02 (60.0\%)

Always

27 (15.9\%)

Never

$41(24.1 \%)$

Sometimes

170

Total

$69(40.6 \%)$

6. Was the cost of travelling there a problem for you?

Fairly

$82(48.2 \%)$

Not at all

19 (11.2\%)

Very

170

Total

7. So, taking all these things above into consideration was travelling for Herceptin treatment overall a problem for you?

Yes

$126(74.1 \%)$

No

44 (25.9\%)

Total

170

8. Did the medical or nursing staff ever have any difficulty giving the Herceptin injection subcutaneously?

Never

159 (93.5)

Sometimes

$9(5.3)$

Very often

2 (1.2)

Total

170

9. How many minutes did it usually take?

$<5$ minutes

$130(76.5)$

6-10 minutes

39 (22.9)

11-15 minutes

$1(0.6)$ 


\section{PATIENT SATISFACTION QUESTIONNAIRE}

Item

Category

Frequency $n(\%)$

10. How painful was this usually?

\begin{tabular}{llc} 
& Fairly & $52(30.6)$ \\
& Not at all & $113(66.5)$ \\
& Very & $5(2.9)$ \\
\hline Total & & 170
\end{tabular}

11. How much were you bothered by any bruising around the SC site?

Never

$115(67.6)$

Somewhat

$52(30.6)$

Very much

$3(1.8)$

170

Total

12. How much were you bothered by any irritation around the SC site?

Never

$129(75.9)$

Somewhat

39 (22.9)

Very much

$2(1.2)$

170

Total

13. How much were you bothered by any infection around the SC site?

Never

$156(91.8)$

Somewhat

$14(8.2)$

Total

170

14. How much were you bothered by reactions to the SC infusion during or directly after it was given?

A little

$38(22.4)$

Never

$129(75.9)$

Very much

$3(1.8)$

170

\section{Total}

$10(24.4)$

Most infusions

$31(75.6)$

Only at 1 st/ 2nd infusions

41

\section{Total}

16. How anxious did having the SC treatment make you feel?

Fairly

$32(18.8)$

Not at all

$128(75.3)$

Very

$10(5.9)$

Total

170

17. In general, how would you describe these SC treatment sessions?

Acceptable

$163(95.9)$

Fairly unpleasant

$7(4.1)$

170

Total

18. In general, how would you describe your experience regarding the Herceptin SC treatment?

Satisfactory

$166(97.6)$

Unsatisfactory

4 (2.4)

Total 
Eur J Breast Health 2022; 18(1): 63-73

\section{Appendix 2. Healthcare Professional Satisfaction Questionnaire}

\section{HEALTHCARE PROFESSIONAL SATISFACTION QUESTIONNAIRE}

Item Category

1. Did you personally administer the SC Herceptin in the study?

\begin{tabular}{lll} 
& Always & $2(12.5 \%)$ \\
& Never & $12(75.0 \%)$ \\
& Sometimes & $2(12.5 \%)$ \\
\hline Total & & 16 \\
\hline
\end{tabular}

2. How many minutes preparation time was required after receiving the Herceptin vial from the pharmacy?

\begin{tabular}{lll}
$<5$ & $7(43.8 \%)$ \\
& $6-10$ & $6(37.5 \%)$ \\
& $16-20$ & $1(6.3 \%)$ \\
& $>20$ & $1(6.3 \%)$ \\
& Not sure & $1(6.3 \%)$ \\
\hline Total & & 16 \\
\hline
\end{tabular}

3. How many minutes in total did it usually take to administer the Herceptin subcutaneously using the handheld syringe?

$<5$

$12(75.0 \%)$

6-15

$4(25.0 \%)$

Total

16

4. How many patients do you think had irritation around the SC site?

\begin{tabular}{lll} 
& A few & $9(56.3 \%)$ \\
& None & $7(43.8 \%)$ \\
\hline Total & & 16
\end{tabular}

5. How many patients do you think had bruising around the SC site?

\begin{tabular}{lll} 
& A few & $3(18.8 \%)$ \\
& None & $13(43.8 \%)$ \\
\hline Total & & 16 \\
\hline
\end{tabular}

6. How many patients do you think had infection around the SC site?

\begin{tabular}{lll} 
& A few & $1(6.3 \%)$ \\
& None & $15(93.8 \%)$ \\
\hline Total & & 16 \\
\hline
\end{tabular}

7. Reactions related to the SC infusion - fever, chills, flu-like symptoms, rash, swelling of lips or face etc. - at time of administration or directly after?

\begin{tabular}{lll} 
& A few & $4(25.0 \%)$ \\
& None & $12(75.0 \%)$ \\
\hline Total & & 16 \\
\hline
\end{tabular}

8. How long do you think the SC sessions usually lasted from patients' arrival until departure?

$\begin{array}{ll}<2 \text { hours } & 1(6.3 \%) \\ >2 \text { but }<3 \text { hours } & 5(31.3 \%) \\ >3 \text { but }<4 \text { hours } & 8(50.0 \%) \\ >4 \text { hours } & 2(12.5 \%)\end{array}$

Total

$16(100.0 \%)$

Not at all 


\section{HEALTHCARE PROFESSIONAL SATISFACTION QUESTIONNAIRE}

Item

Category

Frequency $n(\%)$

10. How reliable was using the handheld syringe to give Herceptin subcutaneously?

Very reliable

$10(62.5 \%)$

Fairly reliable

$6(37.5 \%)$

Total

16

11. Overall, how easy did you/your staff find giving Herceptin subcutaneously using the handheld syringe?

Very easy

$10(62.5 \%)$

Fairly easy

$6(37.5 \%)$

Total

16

12. How likely would you be to offer or recommend SC administration of Herceptin via a handheld syringe to your patients in the future?

Very likely

Fairly likely

$3(18.8 \%)$

Total

16

13. In general, how would you describe your experience regarding the Herceptin SC treatment?

Satisfactory

$16(100.0 \%)$

14. Do you have any other comments to make about the administration of Herceptin during the study?

I think that it is more effective than IV application for patients.

$1(50.0 \%)$

It is time saving for doctors, nurse and patients also it is comfortable for patients

$1(50.0 \%)$ 